\title{
Breast cancer metastasis to the stomach
}

\author{
Francesco D'Angelo1, Alessia Rampini', Silvia Cardella1, Laura Antolino', Giuseppe Nigri', Stefano \\ Valabrega', Paolo Aurello', Giovanni Ramacciato' ${ }^{1}$
}

U.O.C. of General Surgery, "Sapienza" Università di Roma, Sant'Andrea University Hospital, Rome 00189, Italy.

Correspondence to: Dr. D'Angelo Francesco, U.O.C. of General Surgery, "Sapienza" Università di Roma, Sant'Andrea University Hospital, via di Grottarossa 1035-1039, Rome 00189, Italy. E-mail: francesco.dangelo@uniroma1.it

How to cite this article: D'Angelo F, Rampini A, Cardella S, Antolino L, Nigri G, Valabrega S, Aurello P, Ramacciato G. Breast cancer metastasis to the stomach. J Cancer Metastasis Treat 2019;5:30. http://dx.doi.org/10.20517/2394-4722.2018.86

Received:1 Dec 2018 First Decision: 24 Jan 2019 Revised: 1 Feb 2019 Accepted: 10 Feb 2019 Published: 17 Apr 2019

Science Editor: William P. Schiemann Copy Editor: Cai-Hong Wang Production Editor: Huan-Liang Wu

\begin{abstract}
Aim: This study focuses on the stomach as an unusual but not rare site of metastasis of breast cancer.

Methods: We performed a literature search on gastric metastasis from breast cancer searching for reviews from 2000 to 2018 and case reports from 2013 to 2018. We found 11 reviews and 36 case reports and we compared their findings about important aspects of gastric metastasis, such as disease free survival, overall survival, symptoms, endoscopic findings, therapy, histology, and immunohistochemistry.
\end{abstract}

Results: The incidence of stomach as site of metastasis of breast cancer ranges from $5 \%$ to $18 \%$. Reviews and case reports reached similar conclusions about several of the aforementioned aspects: invasive lobular breast cancer (ILC) is mainly responsible for gastric metastases; disease free survival can vary greatly ranging from 0.5 months to 30 years; gastric metastases usually present with non-specific symptoms, even though five patients in case reports were asymptomatic; linitis plastica is the most common endoscopic finding; immunohistochemistry is essential for differentiating primary gastric cancer from metastasis; the preferred treatment is systemic therapy, but surgery is still an option in case of emergency; median overall survival of patients with gastric metastasis from breast cancer is 24 months.

Conclusion: Breast metastasis to the stomach should be considered in any patient suspecting gastric neoplasm previously treated for breast carcinoma, especially if the treated carcinoma was ILC.

Keywords: Gastric metastasis, breast cancer, immunohistochemistry, stomach 


\section{INTRODUCTION}

Breast cancer is the most common malignant tumor in the female population. Different histologies metastatize to different organs ${ }^{[1]}$ : ductal cancer metastatizes mainly to lungs, bones and liver, while lobular cancer has a tendency to metastatize to the gastrointestinal system ${ }^{[2,3]}$. Gastric metastases from breast cancer are not common, but the autoptic incidence is not negligible, and varies from $5 \%$ to $18 \%{ }^{[3,4]}$. Gastric metastases are metachronous in most cases and even in patients with a known history of breast cancer it may be difficult to correlate the two diseases to a common cause, since the gastric disease often presents only several years after the treatment for the breast tumor ${ }^{[5,6]}$. This late manifestation of the disease may lead to erroneous diagnosis in clinical practice and a correct identification of the disease becomes possible only after performing surgery, even though this approach is used only in limited cases. With this study we want to compare current clinical practice found in case reports with the results presented in recent reviews.

\section{METHODS}

We performed a literature search aimed at finding reviews published from January 1st 2000 to October 31st 2018 about gastric metastasis from breast cancer and case reports on the same argument from January 1st 2013 to October 31st 2018. The results presented in the reviews and in the case reports were compared with the aim of analyzing eventual similarity and differences in the findings discussed therein.

Characteristics of the included studies are summarized in Table $1^{[7-17]}$, whereas characteristic of patients from case reports (2013-2018) are reported in Tables 2-4 $4^{[9,14,18-51]}$.

\section{RESULTS}

\section{Part 1. Reviews}

The search for reviews about gastric metastasis from breast cancer was carried out using the combination of words (gastric OR stomach) and (metastasis OR metastases) and (breast) for the PubMed database, using the filter for reviews. Articles written in languages other than English, articles about primary gastric cancer with breast metastasis and about primary breast cancer metastatizing in organs other than the stomach were excluded. The time span considered went from January 1st 2000 to October 31 st 2018 and 11 articles fulfilling the criteria were found as a result of the search. The main aspects of the included studies are summarized in Table 1.

\section{Incidence}

Nine of the articles reported the incidence of gastric metastasis from breast cancer: the incidence ranged from $0.3 \%$ to $35 \%$, with the value $2 \%-18 \%$ reported in 3 articles.

\section{Breast histology}

Nine reviews reached the same conclusion that the most common histological type of breast cancer responsible for gastric metastasis is the lobular cancer. Two of the articles also reported the occurrence of metastasis associated with different types of cancer, with lobular cancer being identified as the cause of metastasis in $83 \%$ of the cases.

\section{Disease Free survival}

Nine of the reviews cited interval time from the appearance of the primary breast cancer to the presentation of gastric metastasis, however the time intervals were not specifically defined, as for example two articles reported "many years" and one article "few months to several years". Other articles reported a median disease free survival of 5 years, but gastric metastasis may occur up to 30 years after the primary tumor. 


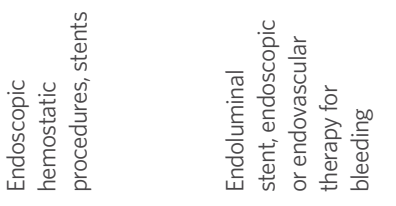

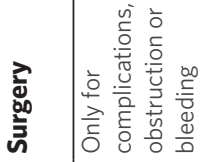

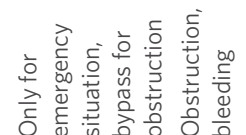
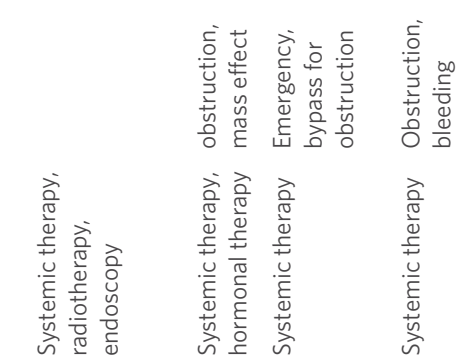

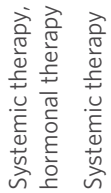

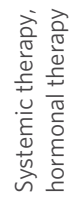

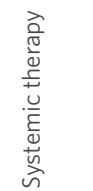

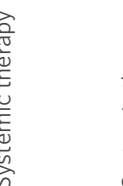

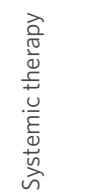

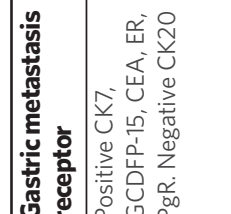

岕离

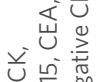

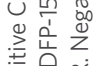

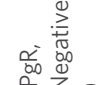

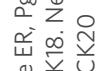

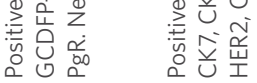

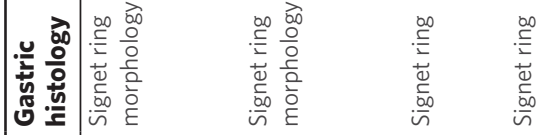

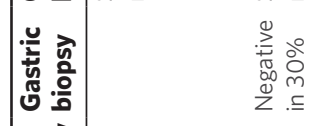

镂

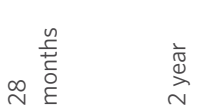

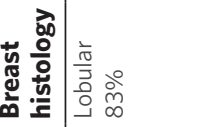

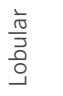

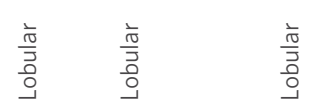

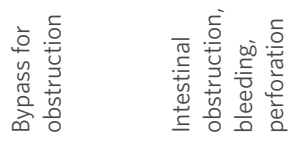

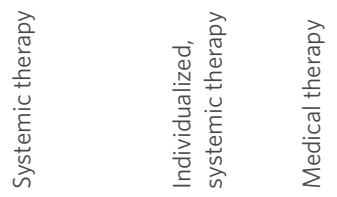

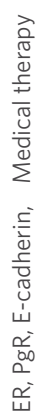

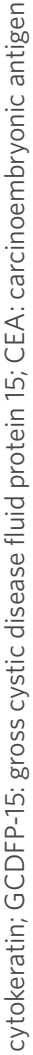

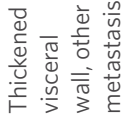

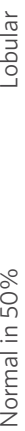

京

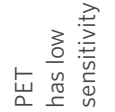

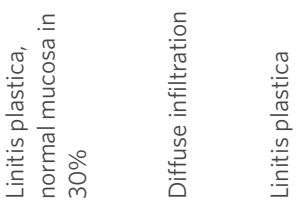

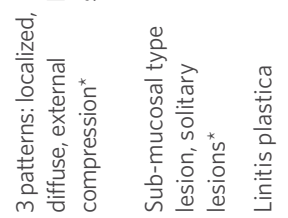

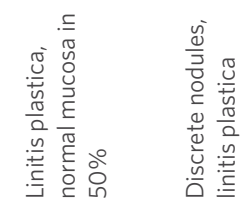

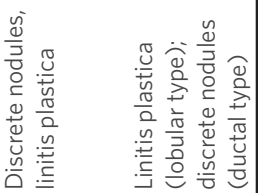

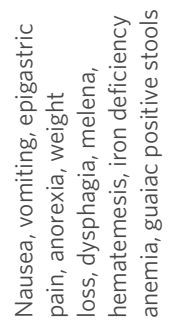

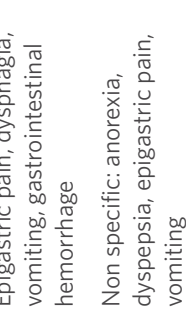

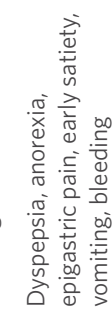

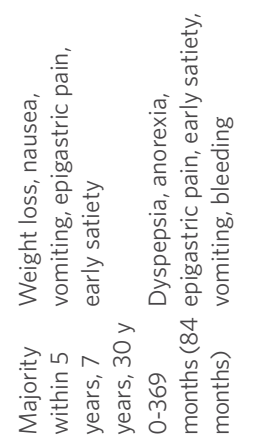

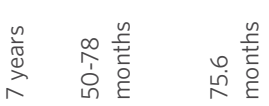

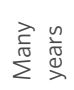

$\frac{\frac{1}{0}}{\frac{0}{3}}$

$\frac{\sqrt{3}}{\frac{10}{3}}$

:

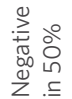


Table 2. Characteristics of patients

\begin{tabular}{lll}
\hline Variables & Number of patients & n (\%) \\
\hline Age, years & 34 & 59 \\
$\geq 55$ & 24 & 41 \\
<55 & & \\
Symptoms & 45 & 90 \\
Yes & 5 & 10 \\
No & & \\
Presentation & 10 & 17 \\
Synchronous & 54 & 83 \\
Metachronous & & \\
Endoscopy & 21 & 37 \\
Linitis plastica & 16 & 29 \\
Ulcers & 4 & 7 \\
Polyp/Nodule & 15 & 27 \\
Other & & \\
Other metastasis & 56 & 81 \\
Yes & 13 & 19 \\
$\quad$ No & & \\
Therapy & 41 & 69 \\
CHT \pm OT & 10 & 17 \\
Surgery + CHT & 7 & 12 \\
Surgery & 1 & 2 \\
RT & & \\
Overall survival & 24 & \\
Median & 23 & \\
CHT & 91 & \\
Surgery + CHT & 45 & \\
Surgery & &
\end{tabular}

CHT: chemotherapy; OT: hormonal therapy; RT: radiotherapy

Table 3. Characteristics of primary breast cancer

\begin{tabular}{lll}
\hline Variables & Number of patients & n (\%) \\
\hline Histology & 42 & 64 \\
Lobular & 16 & 24 \\
Ductal & 8 & 12 \\
Other & & \\
ER status (breast) & 34 & 92 \\
Positive & 3 & 8 \\
Negative & & \\
PgR status (breast) & 29 & 80 \\
Positive & 7 & 20 \\
Negative & & \\
HER2 status (breast) & 7 & 25 \\
Positive & 21 & 75 \\
Negative & & \\
\hline
\end{tabular}

ER: estrogen receptor; PgR: progesterone receptor; HER: human epithelial receptor

\section{Symptoms}

Ten articles reported the most common presentation symptoms related to gastric metastasis: they are often non specific, with epigastric pain as the most frequently reported, followed by nausea, vomiting, dyspepsia and dysphagia. Other reported symptoms are anorexia, early satiety, weight loss, and bleeding that may manifest as hematemesis, melena or iron deficiency anemia. One article used the term "inexpressive" to describe these symptoms.

Endoscopy

All the studies reported the main endoscopic findings, and the majority of them agreed that the most common presentation is linitis plastica. Because linitis plastica is caused by the infiltration of tumor cells in submucosa, the overlying mucosa is normal, thus resulting in negative exam results in $50 \%$ of cases, as reported in two studies, or $30 \%$, as reported by Ayantude. Two articles discussed different patterns of 
Table 4. Characteristics of gastric metastasis

\begin{tabular}{|c|c|c|}
\hline Variables & Number of patients & $n(\%)$ \\
\hline \multicolumn{3}{|l|}{ Gastric Histology } \\
\hline Biopsy & 45 & 80 \\
\hline Definitive specimen & 11 & 20 \\
\hline \multicolumn{3}{|l|}{ ER status (stomach) } \\
\hline Positive & 50 & 91 \\
\hline Negative & 5 & 9 \\
\hline \multicolumn{3}{|l|}{ PgR status (stomach) } \\
\hline Positive & 28 & 61 \\
\hline Negative & 18 & 39 \\
\hline \multicolumn{3}{|l|}{ HER2 (stomach) } \\
\hline Positive & 11 & 30 \\
\hline Negative & 25 & 70 \\
\hline \multicolumn{3}{|l|}{ CK7 (stomach) } \\
\hline Positive & 21 & 91 \\
\hline Negative & 2 & 9 \\
\hline \multicolumn{3}{|l|}{ GCDFP-15 (stomach) } \\
\hline Positive & 9 & 75 \\
\hline Negative & 3 & 25 \\
\hline \multicolumn{3}{|l|}{ CK2O (stomach) } \\
\hline Positive & 1 & 5 \\
\hline Negative & 20 & 95 \\
\hline \multicolumn{3}{|l|}{ MGB (stomach) } \\
\hline Positive & 6 & 86 \\
\hline Negative & 1 & 14 \\
\hline \multicolumn{3}{|l|}{ GATA-3 (stomach) } \\
\hline Positive & 4 & 100 \\
\hline Negative & 0 & 0 \\
\hline \multicolumn{3}{|l|}{ CDX-2 (stomach) } \\
\hline Positive & 0 & 0 \\
\hline Negative & 7 & 100 \\
\hline \multicolumn{3}{|l|}{ E-cadherin } \\
\hline Positive & 3 & 37 \\
\hline Negative & 5 & 63 \\
\hline
\end{tabular}

ER: estrogen receptor; PgR: progesterone receptor; HER: human epithelial receptor; CK: cytokeratin; GCDFP-15: gross cystic disease fluid protein 15; MGB: mammoglobine

presentation, but they analyzed all gastric metastases from other primary tumors and not only the ones related to breast cancer.

\section{Alternative instrumental tools}

Other instrumental tools were not frequently cited. Ayantude and Aurello mentioned that computed tomography (CT) is commonly used to evaluate thickened visceral wall or the presence of other metastases. Namikawa observed that PET has a low sensitivity in diagnosing metastasis, but it may be useful to evaluate response to treatment in those tumors with intense FDG (F-18 fluorodeoxyglucose) uptake.

\section{Gastric biopsy/histology}

Two articles reported that gastric biopsy may be negative in 30\% or even in $50 \%$ of the cases because the tumor cells infiltrate the submucosal layer and not the mucosal layer, which shows a normal histological aspect; there is thus a need for performing deeper biopsy in order to study also underlying layers, which is essential in this particular metastatic pattern. The most common reported histological pattern is signet ring cells, as cited in seven articles, which in some cases may be confused with tumor of gastric origin.

\section{Gastric metastasis receptor}

Immunohistochemistry is essential for differentiating gastric metastasis from primary tumor; positivity for estrogen and progesterone receptors in gastric metastasis is reported in seven articles, and they are the main receptors that direct the diagnosis to a secondary tumor of breast origin. Metastases of breast tumor 
also show positivity for other receptors, such as CK7, CK18, GCDFP-15 and CEA, which are reported in five articles, and they are negative for $\mathrm{CK} 20$ and $\mathrm{CDX}$, which are usually expressed in intestinal cells, and also negative for E-cadherin and HER2, indicating a probable lobular breast cancer origin.

\section{Therapy/Surgery/Palliation}

Ten articles reported that the main therapy for gastric metastasis from breast origin is systemic therapy, either chemotherapy or hormonal therapy; surgery is reserved only in case of complications or emergency, such as bleeding, perforation or obstruction. In this latter case, a more conservative approach is advised and three articles suggest to perform a simple bypass instead of a gastrectomy. Endoscopy can prove very useful and must be considered before surgical approach in case of obstruction and bleeding to put stents or to perform dilatation; also bleeding can be managed conservatively either with endoscopic hemostatic procedures or by embolization radiologists.

\section{Survival}

Only two articles mentioned overall survival and they are approximately concordant, with Jones reporting a survival of 28 months, and Aurello reporting 2 years.

\section{Part 2. Case reports}

We also performed a review of the literature focusing on case reports of gastric metastasis from breast cancer from January 1st 2013 to October 31st 2018 because we wanted to update the aforementioned reviews with the latest current clinical practice present worldwide. We found 36 reports for a total of 69 patients. Data were not reported entirely in all the case reports, so we analyzed and compared only mentioned data. All the patients were females except one male ${ }^{[43]}$. The median age of the patients was 58 years, with a range from 33 to 86 years. For the case reports that reported the age of the patients, 34 (59\%) patients were $>55$ years old, whereas $14(41 \%)$ were $<55$ years.

\section{Breast tumor/receptor}

The most common histological type of breast cancer was ILC (invasive lobular cancer), that was found in 42 cases (63\%); other histological type of breast tumor were IDC (invasive ductal cancer) in 16 (24\%), mixed in $2(4 \%)$, and tubular in 6 cases $(9 \%)$. In one case ${ }^{[30]}$ breast tumor was not found at instrumental research after the diagnosis of gastric metastasis. Breast cancer tumor had ER positive in $34(92 \%)$ cases and negative in 3 (8\%); PgR was positive in $29(80 \%)$ and negative in $7(20 \%)$, and HER2 was negative in 21 (75\%) cases and positive in 7 (25\%). The most common receptor status is ER+/PgR+/HER2-.

\section{Disease free survival}

Usually gastric metastases from breast tumor occur several years after primary cancer; 54 (83\%) patients had metachronous disease, whereas only $10(17 \%)$ had synchronous tumors.

Time of presentation of gastric metastasis from primary breast cancer varies from 0.5 months to 20 years later, with a median time of 61 months (about 5 years). This explains the difficulty to reconduct the gastric disease to breast tumor, because in many cases there was a long time between the manifestations of the two disease.

\section{Symptoms}

Gastric metastasis mostly presented with non specific symptoms in 45 cases, such as epigastric pain (28), nausea and/or vomiting (14), dyspepsia (3), dysphagia (3), anorexia (5), weight loss (12), hematemesis or melena (13), anemia (3). Five patients ${ }^{[24,37,49]}$ were totally asymptomatic, and diagnosis of gastric metastasis in these cases was incidental during exams in follow up. In one case ${ }^{[37]}$ elevated CEA and CA15-3 rose suspiciously for metastasis and then PET scan confirmed gastric localization. 


\section{Endoscopy}

At endoscopy the most common presentation was linitis plastica, present in 21 cases (37\%), whereas ulcers were found in 16 cases $(28 \%)$. In three of these latter cases ${ }^{[27,28,41]}$ the first presentation was perforation, which resulted in elevated morbidity and mortality. Polips or nodules were present in 4 patients (7\%).

\section{Gastric tumor/receptor}

Histological study of gastric metastasis showed in the majority of cases adenocarcinoma with signet ring cells. In six (9\%) patients initial gastric biopsies suspected a primary gastric cancer so they were submitted to surgical treatment; only after histological study of the specimen it was clear that they were metastases from breast cancer and not primary tumor of the stomach.

Immunohistotype is essential to diagnose and differentiate primary gastric cancer from metastatic tumor; the mainly studied receptors were ER, that was positive in 50 (91\%) cases and negative in 5 (9\%); PgR, positive in $28(61 \%)$ cases, and negative in $18(39 \%)$; HER2 negative in 25 (70\%) and positive in $11(30 \%)$; CK7 positive in 21 (91\%) and negative in 2 (9\%) cases; CK20 negative in 20 (95\%) and positive in only 1 case (5\%); CDX2 negative in 7 cases (100\%); GCDFP-15 positive in $9(75 \%)$ and negative in 3 cases $(25 \%)$; GATA-3 positive in 4 cases, Mammoglobine positive in 6 cases $(86 \%)$ and negative in $1(14 \%)$; E-cadherin positive in $3(37 \%)$ and negative in 5 cases $(63 \%)$.

In 45 cases immunohystological study of the biopsies from gastric lesions or gastric wall were taken before starting any treatment; considering patients who underwent gastrectomy, nine ${ }^{[23,26,27,28,35,42,43,45]}$ were taken after surgical treatment whereas three ${ }^{[23,28,43]}$ were done before, but authors decided to perform surgery because they suspected primary gastric cancer or they couldn't exclude it.

In two patients ${ }^{[22,43]}$ immunohistological pattern of gastric metastasis was different from the one of primary breast cancer; in one patient ${ }^{[40]}$ pattern changed during treatment history, passing to a luminal A type and then to a triple negative.

\section{Other metastasis}

Gastric metastasis is usually part of a systemic disease and presents with other localization of malignant cells; considering all case reports, 56 patients (81\%) presented with other metastases at time of diagnosis.

\section{Therapy}

The preferred treatment is systemic therapy, as chemotherapy, endocrine therapy or a combination of both of them; 41 of the patients were subjected to chemotherapy with or without hormonal therapy. Combination of surgery and chemotherapy was used in 10 patients ${ }^{[23,28,29,35,37-39,41,43,45]}$; two of them ${ }^{[28,29]}$ were submitted to neoadjuvant chemotherapy followed by surgery in the belief of a primary gastric cancer. Surgical treatment alone was chosen in $7^{[26,27,42]}$ patients, with three ${ }^{[27,42]}$ of them in an emergency setting for bleeding and perforation. Endoscopic stenting for obstruction was used successfully in 2 patients ${ }^{[19,22]}$.

\section{Survival}

Median overall survival was 24 months, in line with data given by current literature. In some papers survival at follow up was the only data cited, and the media of these data was 28 months. Patients submitted to chemotherapy had an OS of 23 months; patients subjected to surgical treatment alone had an OS of 45 months. Those subjected to surgery plus chemotherapy had an OS of 91 months. We must consider the fact that for this latter group only two patients were considered and that they had stomach as the only site of metastasis. However, due to the paucity of number of cases considered, this data can be misleading.

\section{Part 3. Comparison between reviews and case reports}

The final results from the review and the case reports show that the breast cancer that is responsible for most of the metastasis is the lobular type ( $83 \% v s .63 \%)$. Both summaries report that the time of presentation of 
gastric metastasis is very variable, and ranges from few months to many years (20 years vs. 30 years), and that median disease free survival is 5 years.

Symptoms related to gastric metastasis are reported in both studies as non specific, such as epigastric or abdominal pain, nausea or vomiting, dysphagia, dyspepsia, anorexia, weight loss, and bleeding that may manifest as hematemesis, or melena or anemia. Interestingly, in five patients ${ }^{[24,37,49]}$ of case reports there were no symptoms and diagnosis of gastric metastasis was based on other signs, such as elevated serum markers or incidental findings at instrumental routine follow up.

Regarding endoscopic findings, the principal pattern is linitis plastica in both studies; reviews say that in $30 \%-50 \%$ of cases endoscopic findings are negative, but this aspect is not present in the majority of case reports.

The most common histological pattern found in gastric metastasis is adenocarcinoma with signet ring cells; this pattern can be confused with primary gastric cancer, as said in the reviews; indeed in three case reports ${ }^{[37-39]}$ preoperative findings showed this pattern on gastric biopsies and surgical treatment was performed.

Immunohistochemistry is mainly based on expression of ER and PgR. They are reported as positive in reviews, and, in the case reports, 50 (91\%) tumors had ER positive and 28 (61\%) had PgR positive; other receptors are HER2 and E-cadherin, whose absence is related to lobular cancer (negative in reviews, in case reports 25 (70\%) tumor had HER2- and 5 (63\%) had E-cadherin-); positivity for CK7, GCDFP-15 and MGB and absence of $\mathrm{CK} 20$ and $\mathrm{CDX} 2$ are also related to breast cancer origin. Systemic therapy is the treatment of choice in the reviews and even in case reports (used in 41 patients). Surgery is usually performed in case of complications such as perforation or bleeding; in three cases patients were submitted to emergency laparotomy, two ${ }^{[28]}$ for perforation and septic shock and one ${ }^{[42]}$ for bleeding and hemorrhagic shock. Main surgical intervention is gastrectomy (performed in 13 patients), whereas in one patient ${ }^{[4]}$ only raffia and biopsy were performed in the setting of a perforation. Bypass, that is considered the best option according to reviews, was not even considered in case reports. Indeed obstruction can be managed conservatively by endoscopic stenting as mentioned in reviews, and this was performed in two patients ${ }^{[19,22]}$. No embolization was performed in case reports. Median overall survival is similar (about 2 years) in the two summaries. Unfortunately reviews didn't subdivide OS by type of treatment.

\section{DISCUSSION}

Breast cancer is the most common malignancy in women and the leading cause of cancer-related death in female gender. Global incidence increased about 3.1\% every year in the past 30 years, with an increase of the number of cases in Middle East, south Asia, southeast Asia, and central Latin America, and also mortality increased at an annual rate of $1.8 \%{ }^{[1]}$. Malignant proliferation may arise from ductal or lobular ephitelium: the most frequent is the ductal type that includes $75 \%-82 \%$ of all cases ${ }^{[4,52,53]}$. Other less frequent types are lobular carcinoma (4\%-10\% of all cases), phylloides, or tubular cancer.

Metastases are possible either in ductal and lobular carcinoma, but they may develop in different organs: ductal carcinoma metastatizes more frequently to the lung, the brain and the liver, whereas lobular cancer tends to metastatize to the gastrointestinal (GI) tract, gynecologic organs, peritoneum and bones ${ }^{[2,3]}$. Breast cancer, melanoma and lung cancer, represent the most frequent malignancies metastatizing to GI tract ${ }^{[15,16,53]}$ : the common sites of GI metastasis from breast tumor are colon and rectum (45\%), stomach (28\%), small intestine (19\%), and esophagus $(8 \%)^{[53]}$. Median overall survival of patients with gastric metastasis ranges from 24 to 36 months $^{[53]}$. 


\section{Incidence}

Gastrointestinal metastases from breast cancer are rare, among them the stomach is the second most common site. In McLemore et al. ${ }^{[53]}, 2005$ study, which regarded 12,001 patients, gastric involvement represents $28 \%$ of all cases. The incidence of breast cancer metastasis to the stomach is reported to be from $0.1 \%$ to $6 \%$, but in autopsy series the estimated incidence is found to be higher, from $5 \%$ to $18 \%{ }^{[3,4]}$. Gastrointestinal metastases are usually associated with other concurrent tumor localization: in a retrospective study lead by Taal et al. ${ }^{[3]}, 1992$ up to $94 \%$ of patients present with disseminated disease, that involve the skeleton (60\%), liver $(20 \%)$, and also the lung $(18 \%)^{[4]}$. In our study $81 \%$ of the patients had other metastases at time of diagnosis.

\section{Metastasis}

Gastrointestinal metastasis from breast cancer can be synchronous or more frequently metachronous, with a mean time of presentation reported to be from 6 to 7 years ${ }^{[5]}$, but in literature time can vary from 2 to 30 years $^{[5,6]}$. In our study there were 54 cases with metachronous presentation and only 10 patients with synchronous disease.

Studies report three main mechanisms of dissemination of malignant breast cells to the upper GI tract: lymphatic spread, hematogeneous route, and direct invasion from surrounding organs ${ }^{[3,4,54,55]}$. The first type is more common in esophageal metastasis: the typical presentation is stricture or submucosal nodules with normal overlying mucosa, that makes endoscopic diagnosis difficult ${ }^{[54,55]}$. Also the infiltration of paraesophageal lymphonodes can result in compression of the esophageal lumen with consequent intramural infiltration ${ }^{[56]}$. Hematogenous spread is typical of esophagus, stomach or duodenum which results in stenosis or mass localized intramural or in the submucosal layer, that may become ulcerated. The most common pattern of presentation is linitis plastica-like; in this situation malignant cells are trapped from blood stream to the submucosal or subserosal layer with diffuse infiltration that cause diffuse thickening and rigidity of the organ wall ${ }^{[4,54,55,57]}$.

An important role in metastatic pathway is played by chemokines. CXCR4 and CXCR7 are the main chemokine receptor expressed in breast cancer cells involved in transendothelial migration (TEM), and they are responsible for the chemotaxis to certain target organs ${ }^{[58]}$. CXCR4+ tumors are associated with more distant metastasis than CXCR4-tumors, even though this association is not statistically significant ${ }^{[59]}$. Their ligandsareCXCL12 and CCL21; the first is implied in modulating integrin expression, metalloproteinase production, tumor angiogenesis, tumor cell adhesion and apoptosis ${ }^{[60,61]}$. Metalloproteinases are essential to degrade extracellular matrix to permit invasion of the cells attracted in metastatic sites by chemokines from the bloodstream ${ }^{[60]}$. CXCL12 promotes homing of tumor cells to secondary sites and then recall endothelial stem cells for blood vessel formation and subsequent proliferation ${ }^{[61]}$.

Circulating breast tumor cells pass from blood or lymphatic stream to areas that express CXCL12, thereby metastatizing in many organs such as bone marrow, lymph nodes, liver and lung ${ }^{[59,61]}$.

An interesting hypothesis suggested by an article by J. Carlos Villa Guzman highlights the implication of inflammation response in tumorigenesis. Chronic inflammation induced by Helicobacter Pylori is associated with higher expression of chemokines and interleukins that may attract tumor cells to gastric or colon mucosa with subsequent proliferation and develop of metastatic disease ${ }^{[9]}$. Even though these mechanisms are not fully understood, this hypothesis offers an interesting start point for other future studies.

\section{Lobular breast cancer}

Breast cancer metastases are more frequently related to lobular type then ductal type; in $83 \%$ of all metastases the primary breast cancer has lobular histotype ${ }^{[4]}$. According to the study of Borst et al. ${ }^{[62]}$, 1993 


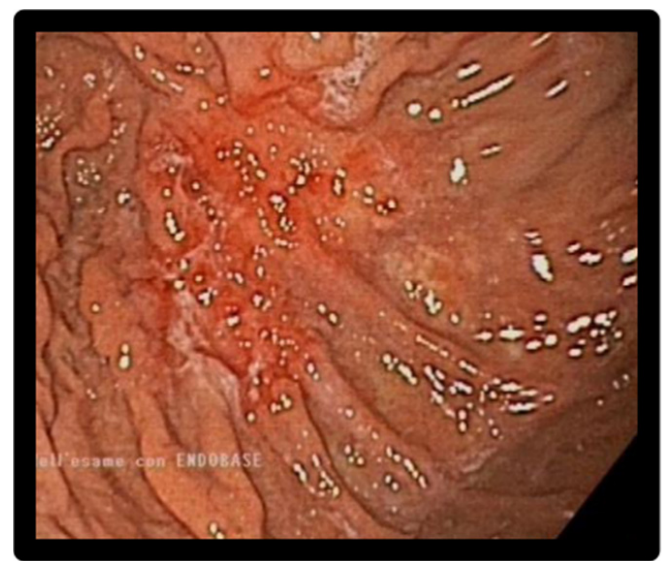

Figure 1. Endoscopy showing localized lesion at gastric corpus. Biopsy confirmed the presence of gastric metastasis from breast cancer

the incidence of lobular carcinoma metastasis is higher in organs such as gastrointestinal system $(4.5 \%$ vs. $0.2 \%$ in ductal carcinoma); gynecologic organs ( $4.5 \%$ vs. $0.8 \%)$; peritoneum-retroperitoneum ( $3.1 \%$ vs. $0.6 \%$ ); bone marrow( $21.2 \%$ vs. $14.4 \%$ ). We found 42 cases with gastric metastasis from lobular carcinoma, whereas 16 cases were related to ductal carcinoma. The propensity of lobular breast cancer to give metastasis seems to be correlated with mutations of E-cadherin genes; the impaired function of the produced protein determines loss of adhesion among epithelial cells. Cells are initially separated from each other so that they can invade the surrounding tissue and then enter in the lymphatic system or in the bloodstream, leading to the progression of metastatic disease ${ }^{[63,64]}$.

\section{Symptoms}

Gastric metastases of breast cancer have no specific symptoms and may often be confused with primary gastric cancer or other conditions, such as effects of chemotherapy, radiotherapy, oral medications, liver metastasis or hypercalcemia ${ }^{[4,57]}$. These symptoms include anorexia, dysphagia, feeling bloated soon after eating, bleeding (melena, hematemesis), dyspepsia, epigastric pain and retch $^{[65,66]}$. Common findings in blood tests are iron deficiency anemia or abnormal high levels of CA 15.3 that would arise the suspect for a relapse of primary breast cancer ${ }^{[9,67]}$. Gastric metastases usually occur many years after primary breast cancer, so when the patient presents with these vague symptoms it's difficult to reconduct them to the primary causative disease. In our case reports, nine ${ }^{[23,28,29,35,37,38,39,43,45]}$ of the patients had a wrong initial diagnosis and they were submitted to surgical treatment in the suspect of a primary gastric cancer.

\section{Diagnosis (instrumental)}

Endoscopy is the main diagnostic tool when patients present with symptoms related to upper GI disease. Gastric metastases from breast cancer show three main different patterns: localized pattern (in about 18\% of cases, as large ulcers and polyps [Figure 1], diffuse infiltration (57\% of cases, such as linitis plastica-like with diffuse infiltration of the submucosal and seromuscolar layer with a fibrotic reaction that causes narrowing lumen, rigidity, wall thickening with reduced peristalsis), and external compression (in $25 \%$ of cases) ${ }^{[4,68,69]}$. Gastric metastases are usually localized in submucosal and seromuscolar layers; in more than $50 \%$ of cases endoscopy study is negative ${ }^{[70]}$. In one case report ${ }^{[18]}$ first histological examination of gastric biopsies was negative; after synchronous diagnosis of primary breast cancer, endoscopy was repeated and biopsies of submucosal layer were performed again, showing gastric metastasis from lobular cancer. Also in another patient ${ }^{[19]}$ initial gastric biopsies were negative, reporting only mild chronic gastritis. Endoscopic ultrasound showed thickening of the muscolaris propria so biopsies were repeated focusing on this layer: muscular wall was infiltrated by malignant cell from lobular breast carcinoma. 

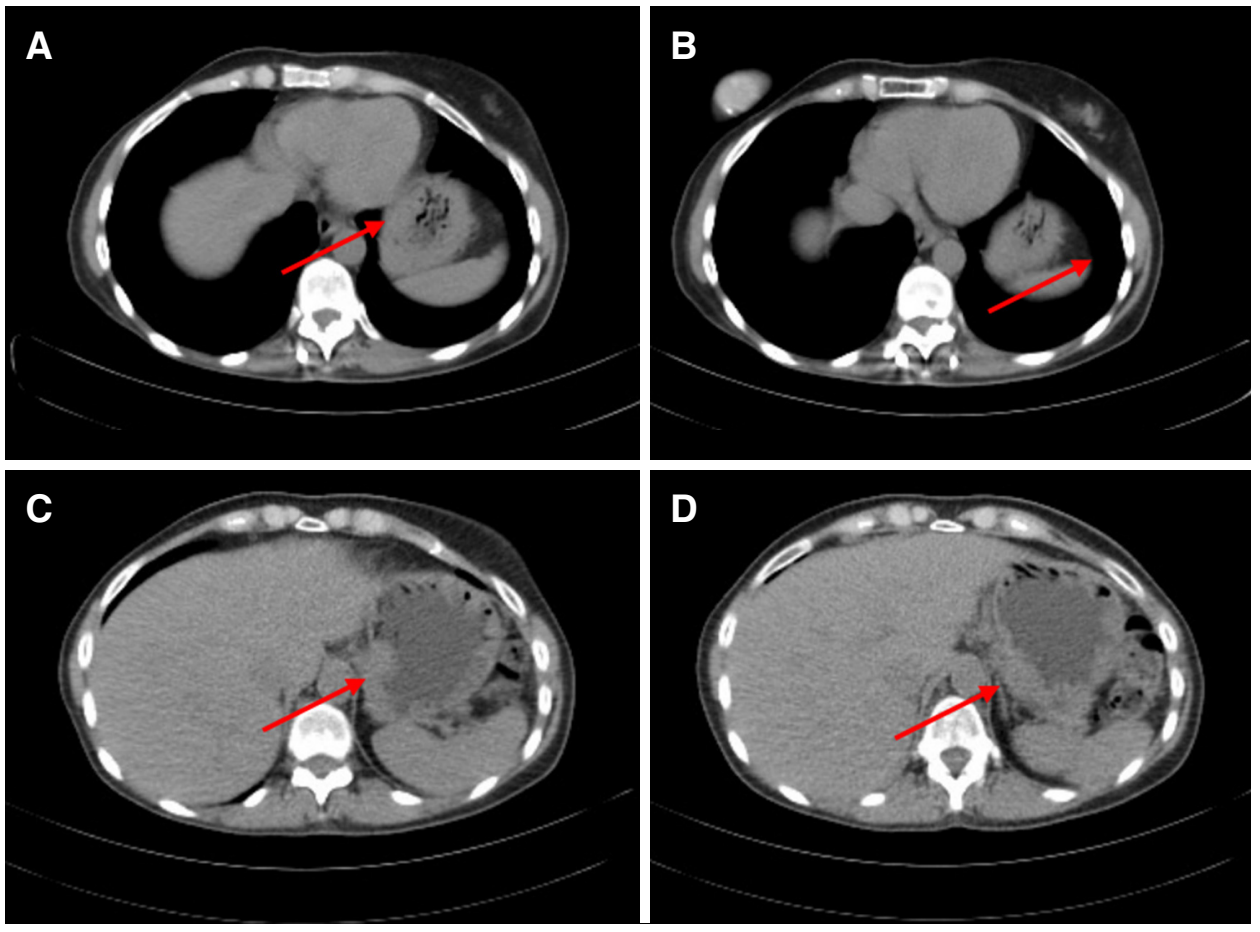

Figure 2. CT scan showing gastric wall thickening at gastric fundus (A-D)

In literature CT scan study is not mentioned as one of the main diagnostic source to establish the nature of primary cancer, but it is used to evaluate wall thickening despite a normal aspect in endoscopic study and other site of metastasis ${ }^{[54]}$ [Figure 2].

A new useful diagnostic approach to differential diagnosis is magnifying endoscopy with narrow-band imaging (ME-NBI); it shows alterations in the framework of microvessels that are characteristic of metastatic pattern in comparison to primary malignancy of the stomach ${ }^{[51]}$.

The sensitivity of PET is lower for the diagnosis of gastric cancer due to physiological absorption of F-18 fluorodeoxyglucose and involuntary movements by the gastric wall [Figure 3]; early cancers, signet-ring cell carcinoma and poorly differentiated non-solid adenocarcinoma are characterized by high false-negative rates. There are also some scenarios of non-specific FDG accumulation correlated to mucosal inflammation, as in superficial gastritis and erosive gastritis, leading to false positives ${ }^{[71]}$.

\section{Histology}

Differentiation of primary gastric cancer from gastric metastasis is crucial; from the histological point of view, the first important difference is the localization of tumor cells: mucosa is generally involved in gastric cancer, while submucosal layer is usually affected in metastatic disease ${ }^{[4,15]}$. In gastric metastasis malignant small cells with monomorphic, round nuclei and vacuolated cytoplasm typically array in chords, named "Indian files", and infiltrate the serosal, muscular and submucosal layer ${ }^{[57]}$.

An additional difficulty is that they share signet ring cell-like morphology, thus lobular metastasis can mimic primary gastric cancer. However breast signet-ring cell carcinoma may show some morphological differences from gastric and colonic signet-ring cell carcinoma. The first shows a single, well-circumscribed univacuolated intracytoplasmatic lumina, with a central eosinophilic inclusion, whereas the latter has an extended, globoid, and optically clear cytoplasmatic acid mucin that pushes the nuclei against the cell membrane ${ }^{[72]}$. 

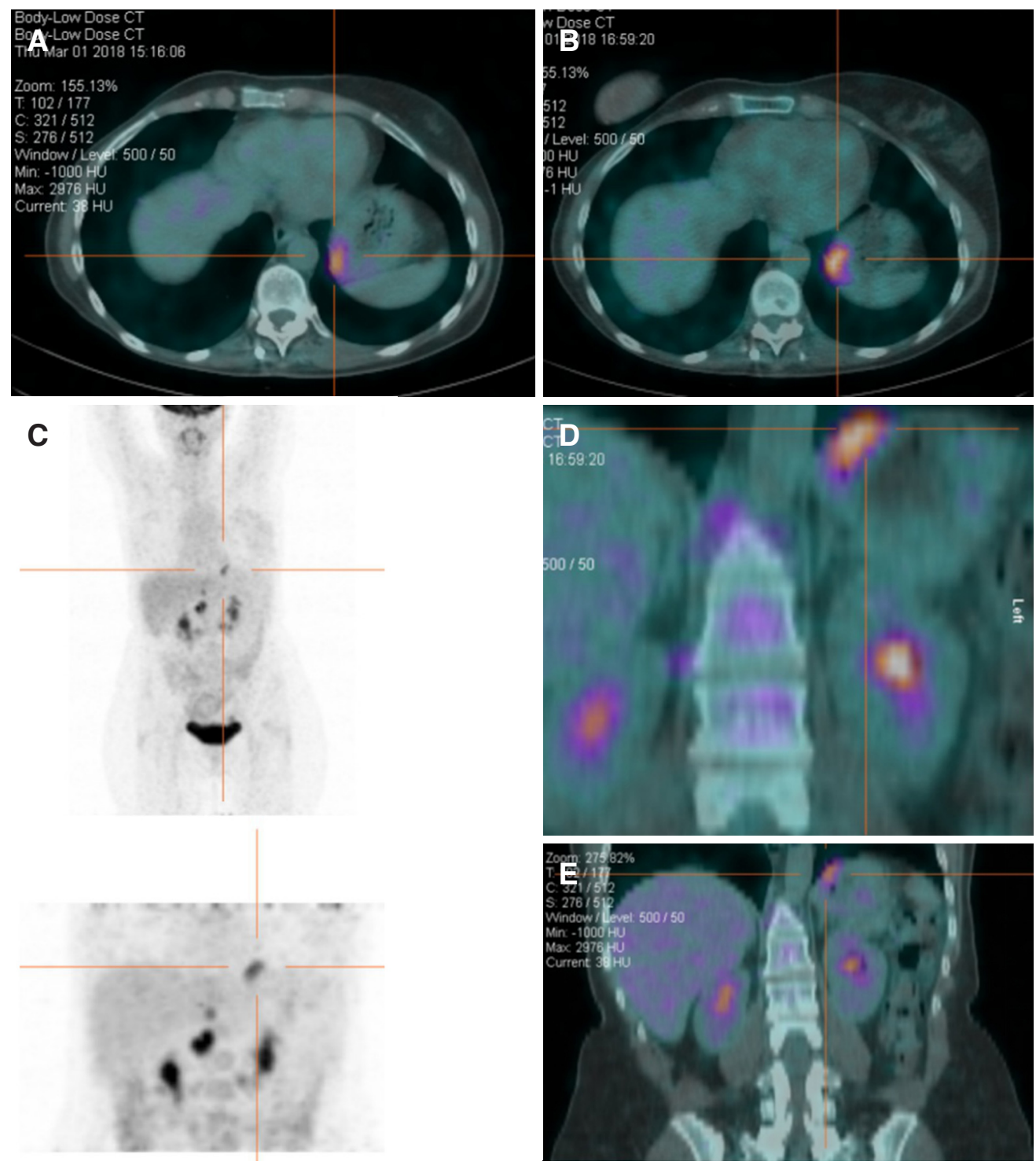

Figure 3. Pet scan showing pathological F-18fluorodeoxyglucoseaccumulation in the gastric fundus (A-E)

Therefore differentiation of primary gastric metastasis requires to evaluate the infiltration of the serosal, muscolar and submuscolar layers by cells that organize in a typical Indian file pattern ${ }^{[57]}$ with a signet ring appearance $^{[73]}$.

\section{Immunohistochemistry}

Immunohistochemical analysis is the most important tool for differentiating between primary gastric cancer and gastric metastasis from breast cancer; the main markers currently employed are estrogen receptor (ER), progesterone receptor (PgR), mammaglobin (MBG), cytokeratin 7 (CK7), cytokeratin 20 (CK20), human ephitelial receptor 2 (HER2), gross cystic disease fluid protein 15 (GCDFP-15), and GATA-3. A new marker is HFN4A, which is discussed below.

The expression of ER and PgR is highly indicative of breast carcinoma metastasis. Primary gastric cancer can express ER in up to $30 \%$ of cases and $\mathrm{PgR}$ in up to $20 \%$ of cases, but also gastric metastasis from breast carcinoma sometimes show negative ER and $\mathrm{PgR}$ rate even if primary breast cancer is $\mathrm{ER}$ and $\mathrm{PgR}$ positive ${ }^{[6,74]}$. Expression of ER, PR and HER2 status can change between primary tumor and metastatic 
lesions in up to $42 \%$ and this change does not correlate with an impairment of overall survival ${ }^{[75]}$ : ER and PR status are different in $14.6 \%$ and $16.7 \%$ of cases respectively, while HER2 changes in about $8.3 \%$ of cases. During treatment it can be useful to take several biopsies to modify chemotherapy or endocrine therapy in the light of ER, Pr and HER2 status ${ }^{[70]}$. Following chemotherapy, in particular anthracycline-based ones, the level of expression of ER, PR and HER2 can change ${ }^{[75]}$; these modifications imply a change also in tumor progression and aggressiveness and a resistance to endocrine therapy or trastuzumab, producing a change in clinical strategy. In our study the male patient ${ }^{[43]}$ had different patterns between gastric metastasis and primary breast tumor (ER-/PgR- in the stomach and ER+/PgR+ in the breast), one ${ }^{[22]}$ had different expression of HER2 (HER2- in the breast, HER2+ in the stomach) and one patient ${ }^{[40]}$ showed a change in receptor status during treatment passing from a Luminal A type to a Luminal B and then to a triple negative.

Combination of $\mathrm{CK} 7 / \mathrm{CK} 20$ is also useful to distinguish between metastasis from breast carcinoma and primary gastric tumor; CK20 is expressed in gastric, colorectal, pancreatic and in transitional cell carcinomas, but it's negative in breast cancer ${ }^{[76]}$. The $\mathrm{CK}_{7+} / \mathrm{CK} 20-$ pattern is typical of adenocarcinoma of the breast, lung, and ovary, while CK7-/CK20+ is expressed in intestinal adenocarcinoma. CDX2 is a tumor suppressor gene that is implied in intestinal cell proliferation, differentiation, adhesion and apoptosis; it's an important marker of intestinal differentiation, usually expressed in gastric cancer and in intestinal metaplasia $^{[77,78]}$.

Mammoglobine (MGB) is a 93-amino acid glycoprotein and a recent marker for breast cancer with a sensitivity of $93.1 \%$. It can be useful in diagnosis of gastric metastasis from breast tumor in combination with other receptor expression ${ }^{[79,80]}$. GCDFP-15 is never expressed in gastrointestinal cancer, but it's found in malignant tumor from the breast, and also salivary gland, external genitals, eyelid, apocrine duct of the bronchial tubes and gynecologic adenocarcinoma ${ }^{[81]}$. MGB is more sensitive than GCDFP-15 but has less specificity ${ }^{[79]}$. GATA-3 is a nuclear transcription correlated to breast glandular epithelial cells and shows the highest sensitivity in comparison to MGB and GCDFP-15 ${ }^{[81]}$.

Diagnosis of metastatic disease stems from the analysis of all these markers. Gastric metastases are usually positive for ER, PgR, mammoglobine, GCDFP-15, GATA-3 and CK7 and negative for CK20. Gastric cancer is normally positive for CK20, but it's usually negative for GCDFP-15, ER and PgR, even if ER and PgR expression is controversial.

HNF4A (hepatocyte nuclear factor 4 alpha) was introduced as a new marker to differentiate gastric metastasis from primary malignant tumor. HNF4A is a nuclear transcription factor correlated with invasion, metastasis and epithelial-to-mesenchymal transition, due to activation of MMP-14 and promoting tumor angiogenesis, migration and invasion ${ }^{[82]}$. A recent Brazilian study shows that HNF4A has positive expression in all patients with primary gastric adenocarcinoma, negative in all cases of primary breast carcinoma and also negative in all gastric metastases from breast carcinoma. In combination with ER and PR expression, it exhibits high sensitivity (100\%) and specificity $(96 \%)^{[83]}$.

\section{Therapy}

Establishing the primary site of malignancy determines the right treatment. Gastric metastasis from breast cancer are considered as a systemic disease because they usually present along with other metastatic localization, therefore the appropriate therapy is systemic, such as chemotherapy or endocrine therapy, whereas for primary gastric cancer first line therapy involves surgery ${ }^{[5,15,84]}$. Chemotherapy, hormonal therapy or a combination of both of them lead to a remission rate of $32 \%$ to $53 \%$ and a prolonged survival of 2 or 3 years $^{[3]}$.

In case of metastatic disease surgery is considered in case of obstruction, bleeding or perforation of gastric wall $^{[85]}$ even if more conservative treatments (endoscopic hemostatic procedures or stent) can be performed 
in patients with low performance status ${ }^{[12]}$. The surgical option must be as little invasive as possible, for example a palliative bypass can be considered in case of outlet obstruction ${ }^{[15]}$.

Many studies, such as the one conducted by McLemore et al ${ }^{[53]}, 2005$ report a lack of survival benefit from surgical treatment; palliative surgery can be associated with a prolonged median survival in some cases, but other factors can affect this difference such as biased patient selection for surgical palliation. In literature, few studies show surgical resection in patients with unique localization with reported improvement in overall survival: the study published by Taal et al. ${ }^{[4]}, 2000$ patients with complete remission of primary breast cancer that underwent gastric resection for solitary gastric metastasis shows a survival time of 38 months to be compared with 14.38 months for patients who didn't undergo resection ${ }^{[3]}$. This can be explained considering that for patients submitted to surgical treatment the stomach is the unique localization without other metastasis or peritoneal carcinomatosis. In these patients the disease is not as advanced as in those candidates for systemic therapy. Therefore enhanced survival is due to patient clinical conditions and not strictly related to the type of treatment. In general, surgery does not offer an increase in survival but may have role in palliation ${ }^{[53]}$. In our study surgery was also used to perform final diagnosis; gastric biopsies were confounding, thus authors performed gastrectomy to rule out the origin of the gastric disease ${ }^{[23,28,29,35,37-39,43,45]}$.

\section{CDH1 mutation}

As mentioned before, E-cadherin is a transmembrane glycoprotein involved in calcium-dependent adhesion; when E-cadherin is mutated, this leads to loss of cell adhesion, cell migration and subsequently tumorigenesis ${ }^{[8]}$. An important gene mutation that is both associated with breast cancer and gastric cancer is $\mathrm{CDH} 1$, that encodes E-cadherin. Families with $\mathrm{CDH} 1$ mutations have a cumulative risk of developing hereditary diffuse gastric cancer (HDGC) of $70 \%$ and $56 \%$ in males and females, whereas female members have a cumulative risk of $42 \%$ for lobular breast cancer by age $80^{[87]}$. Other gene mutations related to breast cancer but not to gastric cancer are BRCA1, BRCA2, and TP53; both BRCA1 and TP53 are associated with invasive ductal carcinoma, BRCA2 with both ductal and lobular carcinoma while $\mathrm{CDH} 1$ is only associated with lobular breast carcinoma ${ }^{[88]}$. $\mathrm{CDH} 1$ is a gene mutation that is mutually exclusive with BRCA1/2 germline mutations. Screening for $\mathrm{CDH} 1$ should be suggested to women who have a personal or a family history of a combination of diffuse gastric cancer and lobular breast cancer (with at least one diagnosed before the age of 50), bilateral lobular carcinoma diagnosed at a young age, or family history of multiple lobular carcinomas with onset before 50 years old without gastric tumour ${ }^{[89]}$. Sometimes diagnosis of lobular breast cancer with early onset might be the first manifestation of HDGC; so in patients with a history of multiple LBCs at a young age, especially with bilateral manifestation, it's advisable to perform a test for gene mutations ${ }^{[90]}$. When $\mathrm{CDH} 1$ mutation is diagnosed, therapeutic management for the stomach and the breast is quite different: usually prophylactic total gastrectomy is advised because of the high risk of DGC, whereas prophylactic mastectomy is not performed, considering various genetic penetrance for LBC, but female patients should undergo to yearly mammography and breast MRI from age 35 years onwards ${ }^{[86]}$.

In conclusion, gastric metastases are a rare but not unusual site of secondarism from breast cancer ${ }^{[3,4]}$. They usually arise several years after diagnosis of primary tumor and sometimes this can mislead the diagnosis ${ }^{[5,6]}$. The differential diagnosis between primary gastric cancer and gastric metastasis is crucial and made possible only by histology and immunohistological patterns. Systemic therapy is the treatment of choice because the disease is usually not only localized to the stomach but presents other concurrent metastases ${ }^{[5,15,84]}$. Surgery still has a role in case of complications or for definitive diagnosis when preoperative biopsy is not diriment and there is still a suspicion for primary gastric cancer ${ }^{[85]}$, even though this latter case is progressively decreasing thanks to innovation in instrumental tools for diagnosis. Breast metastasis to the stomach should be considered in any patient suspected of gastric cancer previously treated for breast carcinoma, especially if the treated carcinoma was ILC. 


\section{DECLARATIONS}

\section{Authors' contributions}

Made substantial contributions to conception and design of the study and performed data analysis and interpretation: D’Angelo F, Rampini A, Cardella S

Performed data acquisition: Rampini A, Cardella S

Performed administrative, technical, and material support: Nigri G, Valabrega $S$

Data review: Antonlino L, Aurello P, Ramacciato G

\section{Availability of data and materials}

Not applicable.

\section{Financial support and sponsorship}

None.

\section{Conflicts of interest}

All authors declared that there are no conflicts of interest.

\section{Ethical approval and consent to participate}

Not applicable.

\section{Consent for publication}

Written informed consent for publication for images used in Figures 1-3 was obtained.

\section{Copyright}

(c) The Author(s) 2019.

\section{REFERENCES}

1. Forouzanfar MH, Foreman KJ, Delossantos AM, Lozano R, Lopez AD, et al. Breast and cervical cancer in 187 countries between 1980 and 2010: a systematic analysis. Lancet 2011;378:1461-84.

2. Fondrinier E, Guérin O, Lorimier G. A comparative study of metastatic patterns of ductal and lobular carcinoma of the breast from two matched series (376 patients). Bull Cancer 1997;84:1101-7.

3. Taal BG, den Hartog Jager FC, Steinmetz R, Peterse H. The spectrum of gastrointestinal metastases of breast carcinoma: I. stomach. Gastrointest Endosc 1992;38:130-5.

4. Taal BG, Peterse H, Boot H. Clinical presentation, endoscopic features, and treatment of gastric metastases from breast carcinoma. Cancer 2000;89:2214-21.

5. Schwarz RE, Klimstra DS, Turnbull AD. Metastatic breast cancer masquerading as gastrointestinal primary. Am J Gastroenterol 1998;93:111-4.

6. Cormier WJ, Gaffey TA, Welch JM, Welch JS, Edmonson JH. Linitis plastica caused by metastatic lobular carcinoma of the breast. Mayo Clin Proc 1980;55:747-53.

7. Pectasides D, Psyrri A, Pliarchopoulou K, Floros T, Papaxoinis G, et al. Gastric metastases originating from breast cancer: report of 8 cases and review of the literature. Anticancer Res 2009;29:4759-63.

8. Ayantunde AA, Agrawal A, Parsons SL, Welch NT. Esophagogastric cancers secondary to a breast primary tumor do not require resection. World J Surg 2007;31:1597-601.

9. Villa Guzmán JC, Espinosa J, Cervera R, Delgado M, Patón R, et al. Gastric and colon metastasis from breast cancer: case report, review of the literature, and possible underlying mechanisms. Breast cancer: target and therapy 2017;9:1-7.

10. Reiman T, Butts CA. Upper gastrointestinal bleeding as a metastatic manifestation of breast cancer: a case report and review of the literature. Can J Gastroenterol 2001;15:67-71.

11. Critchley AC, Harvey J, Carr M, Iwuchukwu O. Synchronous gastric and colonic metastases of invasive lobular breast carcinoma: case report and review of the literature. Ann R Coll Surg Engl 2011;93:e49-50.

12. Weigt J, Malfertheiner P. Metastatic disease in the stomach. Gastrointest Tumors 2015;2:61-4.

13. Namikawa T, Hanazaki K. Clinicopathological features and treatment outcomes of metastatic tumors in the stomach. Surg Today 2014;44:1392-9.

14. Barranco R, Orcioni GF, Ventura F. A fatal gastric perforation secondary to ulcerated metastasis in undiagnosed breast cancer: pathological aspects and review of literature. Malays J Pathol 2017;39:181-7. 
15. Jones GE, Strauss DC, Forshaw MJ, Deere H, Mahedeva U, et al. Breast cancer metastasis to the stomach may mimic primary gastric cancer: report of two cases and review of literature. World J Surg Oncol 2007;5:75.

16. Aurello P, D'Angelo F, Cosenza G, Petrocca S, Stoppacciaro A, et al. Gastric metastasis 14 years after mastectomy for breast lobular carcinoma: case report and literature review. Am Surg. 2006;72:456-60.

17. Ellis MC, Mason T, Barnett J, Kiesow LL, Vetto JT. Gastric malignancies in breast cancer survivors: pathology and outcomes. Am J Surg 2009;197:633-6.

18. Kayılıoğlu SI, Akyol C, Esen E, Cansız-Ersöz C, Kocaay AF, et al. Gastric metastasis of ectopic breast cancer mimicking axillary metastasis of primary gastric cancer. Case Rep Gastrointest Med 2014;2014:232165.

19. Ulmer LL, Cormier I, Jha LK, Singh S, Fisher KW, et al. Use of Endoscopic Ultrasound in a Diagnostic Dilemma: Metastatic Breast Cancer to the Stomach. Case Rep Gastrointest Med 2018;15:2018:2820352.

20. El-Hage A, Ruel C, Afif W, Wissanji H, Hogue JC, et al. Metastatic pattern of invasive lobular carcinoma of the breast-emphasis on gastric metastases. J Surg Oncol 2016;114:543-7.

21. Libânio D, Dinis-Ribeiro M, Pimentel-Nunes P. Gastric metastasis of breast cancer after 20 years. GE Port J Gastroenterol 2018;25:99101.

22. Kim DH, Son SM, Choi YJ. Gastric metastasis from invasive lobular breast cancer, mimicking primary gastric cancer: a case report. Medicine (Baltimore) 2018;97:e0258.

23. Woo J, Lee JH, Lee KE, Sung SH, Lim W. Gastric metastasis as the first presentation one year before diagnosis of primary breast cancer. Am J Case Rep 2018;26:354-9.

24. Horimoto Y, Hirashima T, Arakawa A, Miura H, Saito M. Metastatic colonic and gastric polyps from breast cancer resembling hyperplastic polyps. Surg Case Rep 2018;23:23.

25. Kliiger J, Gorbaty M. Metastasis to the pancreas and stomach from a breast cancer primary: a case report. J Community Hosp Intern Med Perspect 2017;19;234-7.

26. Rodrigues MV, Tercioti-Junior V, Lopes LR, Coelho-Neto Jde S, Andreollo NA. Breast cancer metastasis in the stomach: when the gastrectomy is indicated ? Arq Bras Cir Dig 2016;29:86-9. (in English, Portuguese)

27. Gurzu S, Banias L, Bara T, Feher I, Bara T, Jung I. The epithelial-mesenchymal transition pathway in two cases with gastric metastasis originating from breast carcinoma, one with a metachronous primary gastric cancer. Recent Pat Anticancer Drug Discov 2018;13:118-24.

28. Arslan NÇ, Atıla K, Bora S, Ünlü M. Gastric metastasis of breast cancer mimicking primary gastric cancer: a case report. Turk J Gastroenterol 2012;23:808-9.

29. Buka D, Dvořák J, Richter I, Hadzi ND, Cyrany J. Gastric and colorectal metastases of lobular breast carcinoma: a case report. Acta Medica (Hradec Kralove) 2016;59:18-21.

30. Ulanja MB, Taha M, Al-Mashhadani A, Beutler BD, Al-Tekreeti M, et al. Gastrointestinal bleed from erosive gastritis and duodenitis: a sentinel event of invasive lobular carcinoma of the breast and a diagnostic dilemma. Cureus 2018;10:e2757.

31. Zhang B, Copur-Dahi N, Kalmaz D, Boland BS. Gastrointestinal manifestations of breast cancer metastasis. Dig Dis Sci 2014;59:2344-6.

32. Eren OO, Ozturk MA, Sonmez O, Aslan E, Ozkan F, et al. Gastric metastasis in a patient with lobular breast carcinoma 6 years after diagnosis. J Gastrointest Cancer 2014;45:504-5.

33. Joshi P, Lele V, Jain R, Khubchandani S, Sinhasan S. Stomach metastasis of breast carcinoma mimicking primary gastric neoplasm on fluorodeoxy glucose-positron emission tomography-computed tomography. Indian J Med Paediatr Oncol 2013;34:128-9.

34. Abid A, Moffa C, Monga DK. Breast cancer metastasis to the GI tract may mimic primary gastric cancer. J Clin Oncol 2013;31:e106-7.

35. Fernandes GS, Corrêa TS, Carvalho EP, Katz A, Hoff PM. Gastric and endobronchial metastases in a case of lobular breast cancer. Case Rep Oncol 2013;6:555-60.

36. Tan L, Piao Y, Liu Z, Han T, Song F, et al. Breast cancer metastasis to the stomach confirmed using gastroscopy: a case report. Oncol Lett 2014;8:1205-7.

37. Geredeli C, Dogru O, Omeroglu E, Yilmaz F, Cicekci F. Gastric metastasis of triple negative invasive lobular carcinoma. Rare Tumors 2015;7:5764.

38. Dória MT, Maesaka JY, Martins SN Filho, Silveira TP, Boufelli G, et al. Gastric metastasis as the first manifestation of an invasive lobular carcinoma of the breast. Autops Case Rep 2015;5:49-53.

39. Yagi Y, Sasaki S, Yoshikawa A, Tsukioka Y, Fukushima W, et al. Metastatic gastric carcinoma from breast cancer mimicking primary linitis plastica: a case report. Oncol Lett 2015;10:3483-7.

40. Nakamura J, Okuyama K, Sato H, Yoda Y, Kai K, et al. Repeated changes of the molecular subtype in gastric metastasis from breast cancer: a case report. Mol Clin Oncol 2016;4:695-8.

41. Wong CS, Gumber A, Kiruparan P, Blackmore A. Gastric perforation secondary to metastasis from breast cancer. BMJ Case Rep 2016;2016.

42. Dos Santos Fernandes G, Batista Bugiato Faria LD, de Assis Pereira I, Neves NC, Vieira YO, et al. Gastric metastasis of breast cancer: a case series. Rare Tumors 2016;8:6305.

43. Ricciuti B, Leonardi GC, Ravaioli N, De Giglio A, Brambilla M, et al. Ductal breast carcinoma metastatic to the stomach resembling primary linitis plastica in a male patient. J Breast Cancer 2016;19:324-9.

44. Jmour O, Belaïd A, Mghirbi F, Béhi K, Doghri R, et al. Gastric metastasis of bilateral breast cancer. J Gastrointest Oncol 2017;8:E16-20.

45. Bushan K, Kammar P, Singh C, Advani S, Mahajan P. Infiltrating lobular breast cancer presenting as isolated gastric metastasis: a case report. Idian J Surg Oncol 2018;9:318-22.

46. Khan I, Malik R, Khan A, Assad S, Zahid M, et al. Breast cancer metastases to the gastrointestinal tract presenting with anemia and intraabdominal bleed. Cureus 2017;9:e1429.

47. Namikawa T, Munekage E, Ogawa M, Oki T, Munekage M, et al. Clinical presentation and treatment of gastric metastasis from other malignancies of solid organs. Biomed Rep 2017;7:159-62. 
48. Yim K, Ro SM, Lee J. Breast cancer metastasizing to the stomach mimicking primary gastric cancer: a case report. World J Gastroenterol 2017;23:2251-7.

49. Ushida Y, Yoshimizu S, Horiuchi Y, Yoshio T, Ishiyama A, et al. Clinicopathological features of metastatic gastric tumors originating from breast cancer: analysis of eleven cases. World J Oncol 2018;9:104-9.

50. Xu L, Liang S, Yan N, Zhang L, Gu H, et al. Metastatic gastric cancer from breast carcinoma: a report of 78 cases. Oncol Lett 2017; 14:4069-77.

51. Waseda Y, Hayashi T, Kaneko S. Gastric metastasis from breast cancer visualized by magnifying endoscopy with narrow-band imaging. Dig Endosc 2015;27:708-15.

52. Dupont WD, Page DL. Risk factors for breast cancer in women with proliferative bresat disease. N Engl J Med 1985;312:146-51.

53. McLemore EC, Pockaj BA, Reynolds C, Gray RJ, Hernandez JL, et al. Breast cancer: presentation and intervention in women with gastrointestinal metastasis and carcinomatosis. Ann Surg Oncol 2005;12:886-94.

54. Simchuk EJ, Low DE. Direct oesphageal metastasis from a distant primary tumour is a submucosal process: a review of six cases. Dis Esophagus 2001;14:247-50.

55. Caramella E, Bruneton JN, Roux P, Aubanel D, Lecomte P. Metastases of the digestive tract: report of 77 cases and review of the literature. Eur J Radiol 1983;3:331-8.

56. Varanasi RV, Saltzman JR, Krims P, Crimaldi A, Colby J. Breast carcinoma metastatic to the oesophagus: clinicopathological and management features of four cases and literature review. Am J Gastroenterol 1995;90:1495-9.

57. Tohfe M, Shami P, Aftimos G, Saade M. Gastrointestinal metastases from breast cancer; a case report. South Med J 2003;96:624-5.

58. Zabel BA, Wang Y, Lewén S, Berahovich RD, Penfold ME, et al. Elucidation of CXCR7-mediated signaling events and inhibition of CXCR4-mediated tumor cell transendothelial migration by CXCR7 ligands. J Immunol 2009;183:3204-11.

59. Andre F, Xia W, Conforti R, Wei Y, Boulet T, et al. CXCR4 expression in early breast cancer and risk of distant recurrence. Oncologist 2009; 14:1182-8.

60. Bartolomé RA, Ferreiro S, Miquilena-Colina ME, Martínez-Prats L, Soto-Montenegro ML, et al. The chemokine receptor CXCR4 and the metalloproteinase MT1-MMP are mutually required during melanoma metastasis to lungs. Am J Pathol 2009;174:602-12.

61. Kryczek I, Wei S, Keller E, Liu R, Zou W. Stroma-derived factor (SDF-1/CXCL12) and human tumor pathogenesis. Am J Physiol Cell Physiol 2007;292:C987-95.

62. Borst MJ, Ingold JA. Metastatic patterns of invasive lobular versus invasive ductal carcinoma of the breast. Surgery 1993;114:637-41; discussion 641-2.

63. Hunter KW, Crawford NP, Alsarraj J. Mechanisms of metastasis. Breast Cancer Res 2008;10:S2.

64. Wendt MK, Taylor MA, Schiemann BJ, Schiemann WP. Down-regulation of epithelial cadherin is required to initiate metastatic outgrowth of breast cancer. Mol Biol Cell 2011;22:2423-35.

65. Yu HA, Kim EY, Seo MJ, Chung E, Cho MJ, et al. Stomach and colon metastasis from breast cancer. Ewha Med J 2014;37:98-104.

66. Koike K, Kitahara K, Higaki M, Urata M, Yamazaki F, et al. Clinicopathological features of gastric metastasis from breast cancer in three cases. Breast Cancer 2014;21:629-34.

67. Signorelli C, Pomponi-Formiconi D, Nelli F, Pollera CF. Single colon metastasis from breast cancer: a clinical case report. Tumori 2005;91:424-27.

68. Taal BG, Boot H, van Heerde P, de Jong D, Hart AA, et al. Primary non-hodgkin lymphoma of the stomach: endoscopic pattern and prognosis in low versus high grade malignancyin relation to the MALT concept. Gut 1996;39:556-61.

69. Marshall, ME. Gastrointestinal metastases from carcinoma of the breast. J Ky Med Assoc 1983;81:154-7.

70. Qu Q, Zong Y, Fei XC, Chen XS, Xu C, et al. The importance of biopsy in clinically diagnosed metastatic lesions in patients with breast cancer. World J Surg Oncol 2014;12:93

71. Shimada H, Okazumi S, Koyama M, Murakami K. Japanese gastric cancer association task force for research promotion: clinical utility of ${ }^{18}$ f-fluoro-2-deoxyglucose positron emission tomography in gastric cancer. A systematic review of the literature. Gastric Cancer 2011;14:13-21.

72. Chu PG, Weiss LM. Immunoistochemical characterization of signet-ring cell carcinomas of the stomach, breast and colon. Am J Clin Pathol 2004;121:884-92.

73. López Deogracias M, Flores Jaime L, Arias-Camisón I, Zamacola I, Murillo Guibert J, et al. Rectal metastasis from lobular breast carcinoma 15 years after primary diagnosis. Clin Trasl Oncol 2010;12:150-3.

74. Matsui M, Kojima O, Kawakami S, Uehara Y, Takahashi T. The prognosis of patients with gastric cancer possessing sex hormone receptors. Surg Today 1992;22:421-5.

75. Curtit E, Nerich V, Mansi L, Chaigneau L, Cals L, et al. Discordances in estrogen receptor status, progesterone receptor status, and HER2 status between primary breast cancer adn metastastis. Oncologist 2013;18:667-74

76. Tot $\mathrm{T}$. The role of cytokeratins 20 and 7 and estrogen receptor analysis in separation of metastatic lobular carcinoma of the breast and metastatic signet ring cell carcinoma of the gastrointestinal tract. APMIS 2000;108:467-72.

77. Werling RW, Yaziji H, Bacchi CE, Gown AM. CDX2, a highly sensitive and specific marker of adenocarcinomas of intestinal origin: an immunohistochemical survey of 476 primary and metastatic carcinomas. Am J Surg Pathol 2003;27:303-10.

78. Saad RS, Ghorab Z, Khalifa MA, Xu M. CDX2 as a marker for intestinal differentation: its utility and limitations. World J Gastrointest Surg 2011;3:159-66.

79. Bhargava R, Beriwal S, Dabbs DJ. Mammaglobin vs GCDFP-15: an immunohistologic validation survey for sensitivity and specificity. Am J Clin Pathol 2007;127:103-13.

80. Wang Z, Spaulding B, Sienko A, Liang Y, Li H, et al. Mammaglobin, a valuable diagnostic marker for metastatic breast carcinoma. Int J Clin Exp Pathol 2009;2:384-9.

81. Gown AM, Fulton RS, Kandalaft PL. Markers of metastatic carcinoma of breast origin. Histopathology 2016;68:86-95. 
82. Xiang X, Zhao X, Qu H, Li D, Yang D, et al. Hepatocyte nuclear factor 4 alpha promotes the invasion, metastasis and angiogenesis of neuroblastoma cells via targeting matrix metalloproteinase. Cancer Lett 2015;359:187-97.

83. Jucá PCFC, Corrêa S, Vignal GM, Accioly MTS, Lustosa SAS, et al. HFN4A expression as a potential diagnostic tool to discriminate primary gastric cancer from breast cancer metastasis in a Brazilian cohort. Diagn Pathol 2017;12:43.

84. Sato T, Muto I, Hasegawa M, Aono T, Okada T, et al. Breast signet-ring cell lobular carcinoma presenting with duodenal obstruction and acute pancreatitis. Asian J Surg 2007;30:220-3.

85. Ciulla A, Castronovo G, Tomasello G, Maiorana AM, Russo L, et al. Gastric metastases originating from occult breast lobular carcinoma: diagnostic and therapeutic problems. World J Surg Oncol 2008;6:78

86. Corso G, Intra M, Trentin C, Veronesi P, Galimberti V. CDH1 germline mutations and hereditary lobular breast cancer. Fam Cancer 2016;15:215-9.

87. Gjyshi O, Vashi P, Seewald L, Kohan M, Abboud E, et al. Therapeutic and prophylactic gastrectomy in a family with hereditary diffuse gastric cancer secondary to a CDH1 mutation: a case series. World J Surg Oncol 2018;16:143.

88. Figueiredo J, Melo S, Carneiro P, Moreira AM, Fernandes MS, et al. Clinical spectrum and pleiotropic nature of CDH1 germline mutations. J Med Genet 2019;56:199-208.

89. Corso G, Figueiredo J, La Vecchia C, Veronesi P, Pravettoni G, et al. Hereditary lobular breast cancer with an emphasis on E-cadherin genetic defect. J Med Genet 2018;55:431-41.

90. Benusiglio PR, Malka D, Rouleau E, De Pauw A, Buecher B, et al. CDH1 germline mutations and the hereditary diffuse gastric and lobular breast cancer syndrome: a multicentre study. J Med Genet 2013;50:486-9. 